\title{
Desorption of $\beta$-carotene from mesoporous carbon coated monolith: isotherm, kinetics and regeneration studies
}

\begin{abstract}
b-carotene, a natural antioxidant, beneficial form human health prospective. In this study we had examined the recovery of b-carotene form mesoporous carbon coated monolith (MCCM) by batch process. Desorption kinetics and isotherm studies were carried out by using nhexane as an eluent. Isotherm studies showed better applicability of Langmuir model. The first-order two-compartment three-parameter kinetics model as a function of initial loading concentration and reaction temperature was applied. It was observed that b-carotene desorption process is controlled by both rapid and slow desorption. Results showed that the slow desorption fraction increases from 0.8446 to 0.9007 with increase in initial loading concentration from 10.83 to $39.72 \mathrm{mg} / \mathrm{g}$ while, the slow desorption fraction decreases from 0.9261 to 0.8684 with increase in reaction temperature form 30 to $50^{\circ} \mathrm{C}$. The activation energies for rapid and slow desorption were 7.88 and $44.47 \mathrm{~kJ} / \mathrm{mol}$, respectively. The regeneration studies were carried out by both chemical and thermal process. The regeneration efficiency (RE\%) for the three consecutive cycles reduced from 96.24 to $60.04 \%$ and 94.51 to $81.62 \%$ for chemical and thermal regeneration processes, respectively.
\end{abstract}

Keyword: Mesoporous carbon coated monolith; б-carotene; Desorption; Regeneration 